\title{
Special Issue on Methodological Innovations in Developmental and Life-Course Criminology Research: Editorial introduction
}

\author{
Tara R. McGee ${ }^{1}$ - Paul Mazerolle ${ }^{1}$
}

Published online: 22 March 2016

(C) Springer International Publishing AG 2016

This special issue of the Journal of Developmental and Life-Course Criminology (JDLCC) is dedicated to the importance of advancing methodological innovations on developmental and life-course research on crime and criminal behaviour. Our interest in this topic is both altruistic and self-serving. Altruistic in that we have a collective interest in advancing new forms of methodological innovation in developmental research and research on crime across the life course. Without this our field, our disciplines of knowledge and their attendant methodological procedures will stagnate. Self-serving, in the sense that as developmental and life-course researchers, we are especially pleased to be exposed to and to learn from some of the key debates and innovative methodological approaches that are seeking to push the boundaries of the field. In short, we stand to materially benefit by learning from our colleagues who have contributed to this special issue!

By advancing the first special issue of the JDLCC on methodological innovations, we are not signalling a unidimensional form of innovation for developmental and lifecourse research. Indeed, we see numerous opportunities to showcase other areas of innovation in developmental and life-course research including on theoretical developments, as well as other topic areas (e.g. innovative programmatic responses to criminal behaviour across the life course). Indeed, the expanding depth and intellectual richness of the field of developmental and life-course research provide a number of important opportunities to showcase innovative research that is seeking to advance the field.

Tara R. McGee

tr.mcgee@griffith.edu.au

Paul Mazerolle

p.mazerolle@griffith.edu.au

1 Griffith University, Mount Gravatt, Queensland, Australia 


\section{Methodological Innovation in Advancing Understanding of the Developmental Dimensions of Offending across the Life-Course}

There are numerous ways in which a field of knowledge or inquiry can move forward and advance over time. Uncovering new ideas, making new discoveries, or developing new ways for conceptualizing knowledge represents various ways to advance a field of knowledge. Equally important is the scope to develop and or apply new ways of measuring or analysing key dimensions of social phenomena. In this context, we have put forward this special issue dedicated to methodological innovation on developmental and life-course approaches to understanding offending.

As this area of inquiry has developed, so too has the range of methodological approaches and procedures available to life-course researchers. Given the complexity of developmental and life-course theory and the related empirical questions, the methodological tool kit has expanded over time for researchers keenly interested in understanding the developmental or life-course dimensions of offending behaviour. Not only do researchers have access to increasingly sophisticated tools and techniques to analyse the development and nature of crime and criminal offending, this area of inquiry also gives rise to potential innovations and advances in measuring change in offending over time. Thus, understanding the dynamic processes of offending over time not only provides challenges for methodological innovation but it creates unique windows of opportunity whereby innovation and advances can occur.

In considering the topic for this dedicated special issue, we were cognizant of the significant progress that has been made in the area of developmental and life-course criminology. At the same time, it is important to recognize that stagnation in measurement and methodological procedures and orthodoxy in analytical approaches can significantly impede progress for broadening over understanding of offending patterns across the life course. Thus, to advance understanding, researchers must continue to push boundaries and apply new techniques and approaches for measuring and analysing crime and related factors across the life course.

But, what is truly innovative in advancing new or improved approaches for methodological advancement in understanding crime across the life course? What specific procedures or approaches specifically challenge the status quo?

We believe that a special issue dedicated specifically to methodological innovation allows for the opportunity to both explicate and debate new and existing approaches for measurement and analysis of crime across the life course. Moreover, we do not expect broad agreement over what is or is not innovative in terms of methodological procedures or analyses. However, we see any exercise that advances "boundary pushing" or "boundary re-setting" activities as a necessary and healthy requirement for advancing knowledge about crime across the life course.

\section{Overview of the Special Issue}

Whilst a number of topics are worthy of examination in the area of methodological innovation in developmental and life-course research on crime, we have selected five papers that collectively provide insight into areas of methodological concern and suggested ways forward for developmental and life-course research on crime. 
In the first paper of this special issue, David Greenberg provides a critical review of finite mixture modelling (also known as group-based trajectory analysis or latent class analysis) for analysing crime events, such as arrests, over time. Group-based trajectory modelling has emerged as a very popular method for assessing criminal offending over the life course for a number of years. Greenberg proposes in his paper, titled "Criminal Careers: Discrete or Continuous?", that some less frequently employed alternative methods should also be considered, in light of the limitations of finite mixture modelling. Instead of launching into finite mixture modelling, he argues that multilevel modelling or individual time series approaches are more optimal. Given the widespread use and popularity of the finite mixture modelling procedures, readers will no doubt find this review of the strengths and limitations of the approach, as well as detailed consideration and comparison with other approaches, both informative and compelling.

In their paper "Unpacking the Complexity of Life Events and Desistance: An Application of Conjunctive Analysis of Case Configurations to Developmental and Life Course Criminology", authors Elaine Eggleston Doherty and Jaclyn M. Cwick apply the method of conjunctive analysis of case configurations to life-course criminology. This is a relatively new approach for developmental and life-course criminologists, although it has previously been used successfully to study the convergence of victim, situational, and crime characteristics in predicting criminal events. Their case study for applying this technique is to examine the impact of life events on the desistence process. In doing so, they discovered that conjunctive analysis is a useful technique for exploring the complex interactions that characterize individual lives. Doherty and Cwick also provide a discussion of other applications of the technique for future research, for example, in exploring interactions in social circumstances and also for understanding the interactions of risk and protective factors for offending behaviour.

To complement longitudinal surveys, which often have long gaps between measurement, Amy Anderson, Samantha Clinkinbeard, Timothy Barnum, and Rita Augustyn propose the utilization of burst designs in their paper "Examining Behaviors Using Respondents' Cell Phones and a Burst Design: Drinking and Activities Across the First Year of College Among Transitioning Freshmen". In their research, Anderson and her colleagues demonstrate the way in which regularly contacting participants each month, across 3-day intervals, multiple times per day, provided additional data on developmental processes that was not able to be gathered with the three surveys that they conducted that year, via questionnaire. Whilst these researchers used the method to examine onset of alcohol consumption patterns, they highlight that the method could also be used for many other criminological investigations such as transitions from prison to community, measuring children's after-school activities, and public opinion on criminal justice issues. In short, this methodological technique holds promise for uncovering new information about offending across the life course.

In their article, "Handling Complex Meta-analytic Data Structures Using Robust Variance Estimates: A Tutorial in R", Emily Tanner-Smith, Elizabeth Tipton, and Joshua Polanin provide readers with a step-by-step guide for adopting robust variance estimation when dealing with multiple dependent effect sizes in meta-analyses. In the paper, they provide a demonstration of this approach using a meta-analysis on risk factors for juvenile delinquency. As more research evidence accumulates on various 
topics within developmental criminology and criminology more broadly, it will be necessary for researchers to examine the variability in effect sizes rather than just mean effect sizes. Their tutorial provides the reader with the tools for doing so.

In the final paper for this special issue entitled "Smoothing Group-Based Trajectory Models Through B-Splines", Brian Francis, Amy Elliott, and Mat Weldon explore the use of B-spline smoothers in group-based trajectory models (also known as finite mixture modelling or latent class analysis). This technique is proposed as an alternative to polynomials when estimating the shapes of trajectories. To demonstrate the method, Francis and colleagues present both a simulation study and an analysis of data drawn from the UK Offenders Index. Their analyses showed an overall improvement of the classification rate as well as a more flexible curve shape. As a consequence, the authors have called for the inclusion of this approach in more statistical software packages.

As co-editors of the Journal of Developmental and Life-Course Criminology, we are especially proud of the quality of the papers comprising this first special issue of the Journal. We offer our many thanks to the authors for taking their time to produce such important and innovative work and in considering the Journal as an outlet to showcase their research. Moreover, we thank the many reviewers who dedicated their time and insights into the review process. Clearly, the finished papers have been materially improved by the expert insights of the reviewers who we were privileged to call upon.

In advancing this topic of methodological innovations in developmental and lifecourse research on offending across the life course, as the inaugural topic for a special issue of the Journal of Developmental and Life-Course Criminology, our goals have been threefold. We have been keenly interested in including papers that confront some of the major issues affecting or influencing developmental and life-course criminology researchers, that challenge some of the existing approaches or orthodoxies employed for examining developmental and life-course processes influencing offending, and, finally, in illustrating new ways of producing knowledge to inform further understanding about the development of offending across the life course. Whilst it is ultimately for others to judge, we believe the collection of papers included in this inaugural special issue not only meet these goals but also go some way toward stimulating further debate and discussion about the importance of methodological innovation and advancements in examining and understanding the developmental dimensions of offending across the life course.

Tara Renae McGee

Paul Mazerolle

Co-editors 